WIENER SLAVISTISCHES JAHRBUCH, Band 53/2007, 55-66

(C) 2007 by Österreichische Akademie der Wissenschaften, Wien

R A D O L A V K A T I Č I Ć

\title{
Zur Polemik von Primus Truber mit Paulus Skalich
}

\begin{abstract}
Trubarjev polemični odgovor na anonimno ekspertizo iz leta 1560, v kateri je bilo glede jezika njegovega slovenskega prevoda biblije izrečeno odklonilno mnenje, avtor pa je bil po vsej verjetnosti v Zagrebu rojeni Paulus Skalich, implicitno precej jasno pokaže, da sta bila slovenski in hrvaški knjžni jezik v tem času že dve različni enoti, da je bilo njuno območje veljavnosti povsem neodvisno tako od tedanjih predstav o jeziku in narodnosti kakor tudi od tedaj običajnih poimenovanj in da sta bila jezika že razmejena, kot sta razmejena še danes. Pri tem se pokažejo tudi razlike med dvema predstavnima svetovoma jezika in dvema presojama izraznih vrednot, temelječih na različih izročilih.
\end{abstract}

In der Geschichte des slowenischen und kroatischen protestantischen Drucks wirft ein in seiner vollen Bedeutung bisher wenig beachtetes Detail aufschlussreiches Licht auf eine Frühphase der Beziehungen der kroatischen zur slowenischen Schriftsprache (vgl. Katičić 1997: 123-132).

Im Jahr 1559, irgendwann gegen sein Ende, als Primus Truber gerade dabei war, mit seiner Unternehmung der Herausgabe einer kroatischen Übersetzung des Neuen Testaments aus der Feder seiner glagolitisch gebildeten Mitarbeiter Stefan Consul dem Istrier und Anton dem Dalmatiner gut voranzukommen, sah er sich in seiner Arbeit am Druck slowenischer und kroatischer protestantischer Bücher durch eine ernste Beschuldigung schwer gefährdet. Er wurde nämlich bei seinem Förderer und Beschützer, dem Herzog Christoph von Württemberg, mündlich und schriftlich angezeigt, dass die slowenischen Bibelübersetzungen, die er angefertigt, herausgegeben und ausgestattet hatte, nicht rechtgläubig wären. Darüber hat er am 2. Jänner 1560 an den König von Böhmen Maximilian, den späteren deutschen Kaiser, nach Wien geschrieben und darum ersucht, dass seine slowenischen Bücher auf ihre Rechtgläubigkeit hin überprüft würden. Über seine Lage sagt er dabei Folgendes:

Es ist meinem gnedigen fursten vnd herren von Wuertenberg etc. schrifftlich und mündtlich, nit waiß jch durch wen, furkhomen vnnd angezaigt worden, das jn meinen außgegangnen getruckhten windischen buechern vnrechte dolmetschungen, große jrthumben, falsche außlegungen, schwermerische vnd zwinglische opinionen von der tauff, des herren nachtmal vnd von der justification sein sollen etc., vnd von deß wegen werden meine buecher bey den Windischen mehr schaden vnd vnraths dan nutz schaffen und anrichten. (Elze 1897: 35-36; Rajhman 1986: 38 und 41) 
Durch diese Bezichtigung war Trubers schriftstellerische Tätigkeit ernsthaft bedroht und auch sein Wirken als lutheranischer Prediger und Pfarrer in Kempten war in Frage gestellt. In dieser Drangsal wandte er sich an Baron Hans Ungnad, einen Kärntner Adeligen, der mit hohen Ämtern in der Steiermark, Kroatien und am Hof des Königs Ferdinand bekleidet gewesen war und großes Interesse für die Verbreitung der reformatorischen Kirchenlehre unter den Südslawen gezeigt hatte. Zu der Zeit weilte er am Hof des Herzogs Christoph als sein Berater. Er hat davor alle Ämter in österreichischen Erbländern und Kroatien aufgegeben und ist nach Württemberg gezogen, um seinen protestantischen Glauben frei ausüben zu können. Ungnad hat ihm bereitwillig geholfen, sich vor Herzog Christoph zu rechtfertigen und unter seiner Schirmherrschaft sein literarisches Werk fortsetzen zu können. Zu diesem Zweck musste jedoch ein wohl unterrichteter und der slowenischen Sprache mächtiger Begutachter gefunden werden, der lutheranische Rechtgläubigkeit der biblischen Übersetzungen Trubers und der ihnen beigefügten Texte maßgeblich beurteilen konnte. Dafür wandten sie sich an König Maximilian in Wien, der der Reformation wohlgesinnt war und auch in der Lage, einen solchen Kenner leichter zu finden. Und in der Tat kam schon nach einem Monat ein anonymes lateinisch geschriebenes Gutachten an, in dem Trubers Rechtgläubigkeit vorbehaltlos anerkannt wird, seine Sprache und Graphie aber ernsthaft bemängelt werden. Herzog Christoph war nur an der theologischen Korrektheit von Trubers Arbeit interessiert und hat daher umgehend die Fortsetzung seines Drucks gestattet und nur nebenbei von ihm verlangt, auf Sprache und Schreibung zu achten und die Fehler, die ihm vielleicht unterlaufen wären, auszubessern. ${ }^{1}$

Für uns sind die Einwände, die der anonyme Begutachter gegen Trubers Sprache und lateinische Schreibung erhoben hat, von besonderem Interesse. Es ist wohl am besten, hier nach dem Text seines Gutachtens zu greifen, der uns durch eine glückliche Fügung erhalten ist. Das Schriftstück ist mit Februar 1560 datiert und beginnt folgendermaßen:

Tota continentia libri hujus sclavonici majoris, translatio nempe evangeliorum vera et sincera, cum praefationibus praecedentibus et postillis sequentibus, est sancta et christiana, lingua tamen ipsa seu prolocutio, quam auctor sclavonicam posuit, est quidem sclavonica, tamen arctata et constricta ad illam saltem sclavonicae linguae proprietatem, qua provinciae Styria, Carniola et Carinthia utuntur, ita quod si ista translatio legatur coram illis Sclavis, qui in partibus regni Ungariae superioribus, in comitatibus Trinchiniensi, Arwa, Lypto et aliis vicinis morantur, parum aut fere nihil sint intellecturi. Itidem de Polonis, Bohemis, Moravis, Russis, Muscovitis, Illiricis et his, qui circa Zagrabiam sunt, sentiendum, quod videlicet translatio haec ab illis non intelligatur.

Der ganze Inhalt dieses größeren slawischen Buches, nämlich eine wahre und getreue Übersetzung der Evangelien mit vorangehenden Vorworten und darauffolgenden Postillen ist heilig und christlich, jedoch die Sprache oder Ausdrucksweise selbst, die der Verfasser als slawisch vorgestellt hat, ist zwar slawisch, aber eingeengt und eingeschränkt auf nur jene Eigenheit der slawischen Sprache, welche die Länder Steiermark, Krain und Kärnten

${ }^{1}$ Vgl. Bučar 1910: 140-142, Rupel 1965: 124-127, Rotar 1988: 67-88. 
verwenden, sodass, wenn diese Übersetzung jenen Slawen, die in den oberen Teilen des Königreichs Ungarn, in den Komitaten Trencsen, Arva, Lipto und anderen benachbarten verweilen, vorgelesen würde, diese wenig oder fast nichts verstehen könnten. Das gleiche ist von den Polen, Böhmen, Mährern, Russen, Moskoviten, Illyrikern und denen, die um Agram (Zagreb) herum sind, zu halten, nämlich, dass diese Übersetzung von ihnen nicht verstanden würde. (Elze 1897: 57)

Wenn der Leser sich in diesem Wortschwall des anonymen Begutachters einmal zurechtgefunden hat, wird ihm klar, dass dieser eigentlich Truber vorwirft, das Evangelium ins Slowenische übersetzt zu haben! Offenbar meint der Rezensent, dass eine solche sprachliche Stilisierung für ein Wirken in die Weite des slawischen Sprachraums nicht geeignet ist. Es ist natürlich eigenartig, dass er dabei mit den Slowaken beginnt und ihnen dann die anderen westslawischen sowie die ostslawischen Völker hinzufügt und erst am Ende zu den Truber am nächsten stehenden illyrischen Slawen kommt. Das ist eine Sprachkonzeption, die der Trubers entgegengesetzt ist, der seine Schriftsprache ausdrücklich und konsequent nach der in Krain gesprochenen Volkssprache ausgerichtet hat. Darüber hat er im Vorwort zum Ersten Teil des Neuen Testaments (Tübingen 1557) Folgendes geschrieben:

Vnd von deswegen / bin ich schlecht bey Bewrischer Windischer sprach / vn wie mans auff der Rastzhitz redet / da ich geboren bin / bliben / Vnd vngewonliche vnd Crobatische wörter darein nicht mengen / auch newe nicht wöllen erdichten. (Sakrausky 1989: 105)

Der Begriff der kroatischen Sprache ist für Truber mit der glagolitischen kirchenslawischen Tradition verbunden. Daher ist auch hier, wenn er sagt, dass er in seine Übersetzungen keine „ungewöhnlichen und kroatischen Wörter“ mengt, zunächst an den kirchenslawischen gottesdienstlichen Wortschatz zu denken, dessen Verwendung sehr wohl beim Ausbau der slowenischen Kirchensprache in Frage kam, Truber aber wollte auch im Wortschatz strikt bei seiner volkssprachlichen Grundlage bleiben.

Die Argumentation mit allen slawischen Sprachen, den westlichen, den östlichen und den südlichen, was immer man sonst von ihr halten mag, wirkt frivol. Sie bleibt jeder Wirklichkeit fern, ist leichtsinnig unseriös. Aber auch so ist in ihr der Geist des humanistischen Slavismus zu erkennen, der seinen vollen Ausdruck in der Rede von der Herkunft und dem Fortgang der Slawen (De origine successibusque Slavorum) des Vinko Pribojević (Vincentius Priboevius) aus Hvar - gehalten 1525 in Hvar und 1532 in Venedig im Druck veröffentlicht - bekommen und einen starken Einfluss auf die gelehrten Slawen jener Zeit ausgeübt hat. Aus dieser Sicht zeigen sich alle slawischen Sprachen als nur eine Sprache und alle slawischen Völker als nur ein Volk. Erst vor diesem Hintergrund wird die Argumentation des Anonymus verständlich, sonst erscheint sie grotesk. ${ }^{2}$

Besonders interessant ist wiederum, dass der anonyme Begutachter zu jenen Slawen, die Trubers Übersetzung nicht verstehen werden - zu allerletzt und dadurch

${ }^{2}$ Vgl. Rotar 1988: 71-72. Hier wird die Groteske stark hervorgehoben, der humanistische Sinn jedoch, der dahinter steht, überhaupt nicht beachtet. 
besonders hervorgehoben - ganz ausdrücklich auch jene aus der Umgebung von Agram zählt, also die kajkavisch sprechenden Kroaten. Damit sondert er sie ganz eindeutig vom slowenischen sprachlichen Ganzen ab. Er schenkt ihnen dadurch große Aufmerksamkeit und stellt sie auf die gleiche Ebene mit den Sprechern anderer slawischer Sprachen, so wie er diese aufgezählt hat. Damit zeigt er, dass er über die kajkavischen Kroaten viel weiß.

Nachdem er ein so negatives Urteil über Trubers Auswahl der mundartlichen Grundlage für seine Schriftsprache gefällt hat, geht sein anonymer Begutachter zu einzelnen Einwänden über:

Quin proprietates etiam ipsius Styriacae, Carniolae et Carinthiae linguae tam obscure sunt redditae, ut vulgus seu communis populus illarum provinciarum absque cognitione linguae germanicae non sit intellecturus complura hic posita vocabula, eo quod non pauca sint, quae germanicam potius quam sclavonicam linguam sapiant, prout sunt illa: vrshah, gnada, ferdammene, trosht, nuz, leben, erbszhina, son, aydi, styma, et alia multa, quae longe praestaret per totum opus ita revidere et reddere, ut etiam absque germanicae linguae cognitione quilibet illius linguagii homo possit intellegere ea quae dicantur, hoc namque modo talis haec translatio fieret cum majore fructu et commodo plebis christianae.

Aber auch die Eigenheiten der steirischen, krainerischen und kärntnerischen Sprache selbst sind so dunkel wiedergegeben, dass die Menge oder das gemeine Volk jener Provinzen viele dort verwendete Wörter ohne die Kenntnis der deutschen Sprache nicht verstehen wird, weil ihrer nicht wenige sind, die eher nach deutscher denn nach slawischer Sprache schmecken, wie es diese sind: uržah, gnada, ferdammene, trošt, nuc, leben, erbščina, zon, ajdi, štima und viele andere, die es viel besser wäre, im ganzen Werk erneut durchzusehen und zu übersetzen, sodass auch ohne Kenntnis der deutschen Sprache ein jeglicher Mensch jener Sprache das, was gesagt wird, verstehen könnte, denn auf diese Weise würde eine solche Übersetzung mit mehr Nutznießung und Vorteil für das christliche Volk hergestellt. (Elze 1897: 57)

Da hat der Anonymus Trubers volkstümliche Fremdwörter beanstandet, Germanismen, die zu jener Zeit den Krainer Mundarten eigen waren. Seinem Sprachempfinden waren sie offenbar fremd, er glaubte, dass sie das Verständnis der Übersetzung erschwerten und zeigt damit, dass er Trubers Sprachauswahl überhaupt nicht verstand. Dieser lehnte nichtslawische Wörter gar nicht ab, wenn sie nur volkssprachlich und fest verwurzelt waren (vgl. Rotar 1988: 71-72). Im Hintergrund des vom anonymen Begutachter erhobenen Einwands steht eine ganz anders ausgerichtete Sprachkultur, eine, die Fremdwörter ablehnt, Wörter hingegen, die ausgehend von der Muttersprache etymologisiert werden können, auch dann als zulässig empfindet, wenn sie ungewöhnlich sind und, um verstanden zu werden, eine bestimmte Anstrengung erfordern. Eine solche Grundhaltung ist jedoch Merkmal der kroatischen Sprachkultur, seit dem Kirchenslawischen bis zu unserer Zeit. Später haben die Slowenen Trubers diesbezügliche Einstellung aufgegeben und sich eine neue zu eigen gemacht, die der kroatischen näher kommt.

In der Fortsetzung seines Gutachtens bemängelt der Rezensent Trubers Schreibung mit lateinischen Buchstaben. Um seine Meinung zu bekräftigen, dass dieser in dieser Hinsicht keine wohl angebrachte Lösung gefunden hat, schreibt er weiter: 
In ipsa etiam stampa seu appositione et impressione caracterum exprimentium ipsam linguam sclavonicam mirum quam multa passim inveniantur, imo plena sunt omnia, ubi literae ipsae alphabeticae seu caracteres ita ponuntur, ut si ita exprimantur, non sint satis expressivi et non bene sonent linguam eandem, prout exempli gratia fere semper ponitur litera $\mathrm{z}$, ubi prolatio non debet fieri in ea subtilitate sicut sonat $\mathrm{z}$, sed potius grossum $\mathrm{c}$ cum subsequente $\mathrm{h}$, ut si exprimatur sclavonice homo, non debet dici cum $\mathrm{z}$ zhlouik, prout ipse impressit, sed chlouik, itidem in nomine pater non ozhak, sed ochak ponendum esset; item non luzh, sed luch, quod est lux, zachetak, non sazhetak, principium, chezt, non zhest, nam et hoc valde frequens ponitur, litera videlicet $\mathrm{s}$ loco $\mathrm{z}$; scribendo enim cor non serze, sed szercze ponendum foret, item zapouid, quod est mandatum, non sapuuid, zemlija terra, non semla; item Lucae XI non: sa uolo kir ie nega periatel, sed: za uoliju kir ie nijega priatel quia amicus ejus est. Hoc passim ponitur o loco u et e diverso; non modri sapiens, sed mudri, qualia sunt multa admodum.

Auch im Druck selbst oder in der Nebeneinanderstellung und dem Ausdruck der Buchstaben, welche die slawische Sprache selbst ausdrücken, ist es ein Wunder, wie Vieles gefunden werden kann - alles ist geradezu voll davon - wo die alphabetischen Lettern selbst oder Buchstaben so gesetzt werden, dass sie, wenn sie ausgesprochen werden, wie sie gesetzt sind, nicht genug ausdrücklich sind und nicht richtig nach dieser Sprache lauten, so wie zum Beispiel der Buchstabe $\mathrm{z}$ dort, wo die Aussprache nicht so dünn ist, wie das z lautet, sondern ein dickes $\mathrm{c}$ mit folgendem $\mathrm{h}$, wie wenn man das slawische Wort für Mensch ausspricht, dieses nicht mit z als zhlovik, wie er es gedruckt hat, sondern chlovik, ebenso in der Benennung für den Vater nicht ozhak sondern ochak zu setzen wäre, ebenso nicht luzh sondern luch, was Licht bedeutet, zachetak und nicht sazhetak, Anfang, chezt und nicht zhest, den auch das wird dort sehr oft gesetzt, nämlich der Buchstabe s anstatt von z; beim Schreiben von Herz sollte nicht serze sondern szercze gesetzt werden, ebenso zapouid, was Befehl bedeutet, nicht sapuuid, zemlija Erde, nicht semla; ebenso Lukas XI nicht: sa uolo kir ie nega periatel sondern za uoliju kir ie niega priatel, weil er sein Freund ist. So wird überall o anstatt u gesetzt und umgekehrt. Es ist nicht modri weise sondern mudri, wovon es viel gibt. (Elze 1897: 57-58)

Hier beanstandet der Begutachter sehr entschieden die lateinische Schreibung, die Truber für die slowenische Sprache einführt. Er hält sie für ganz ungeeignet und glaubt immer zu wissen und sagt es auch sehr bestimmt, wie sie sein sollte. Trubers Verwendung der lateinischen Buchstaben für die Schreibung der slowenischen Sprache ist seiner Meinung nach undeutlich, weil sie zu einer falschen Aussprache führen. Der Rezensent glaubt zu wissen, wie die Buchstaben $z$ und $c h$ ausgesprochen werden, nämlich wie heute $z$ und ć und nicht zum Beispiel wie $c, k$ oder $k h$, so wie diese Buchstaben in deutscher Graphie gelesen wurden. Es steht für ihn ebenfalls fest, dass der Buschstabe $s$ nicht wie heute gelesen wird und dass die Laute $s$ und $c$ mit je zwei Buchstaben, wie $s z$ und $c z$, geschrieben werden sollen. Wenn man das anders schreibt, trübt das nach seiner Meinung die Deutlichkeit der Schreibung. Offensichtlich tritt der Begutachter, ohne es ausdrücklich zu sagen, im Namen einer bereits gefestigten und auf eine slawische Sprache angewandten lateinischen Schreibung auf. Seinen Beispielen ist zu entnehmen, dass es eine war wie die im ungarischen Schrifttum traditionell verwendete. Deren Anwendung beim Schreiben in einer slawischen Sprache erschien dem Rezensenten selbstverständlich, und er hat eine solche Schreibung auch für die slowenische Übersetzung biblischer Texte verlangt, als Truber sich daran machte, eine solche anzufertigen.

Weiter behandelt der anonyme Begutachter einige seiner Meinung nach die Glaubenslehre und Seelsorge betreffende Mängel in Trubers Vorworten und Postil- 
len. Da dies mit der Sprache nichts zu tun hat, werden wir uns hier dabei nicht aufhalten. Der abschließende Satz des Gutachtens jedoch, geradezu das ceterum censeo seines Verfassers, ist hier von besonderer Bedeutung. Er schließt seine Ausführungen mit den Worten:

Vellem tamen ex animo videre croaticam illam translationem do. Stephani Consul, de qua hic in praefatione fit mentio.

Ich wünschte jedoch aus ganzer Seele, jene kroatische Übersetzung des Herrn Stephan Consul zu sehen, die hier im Vorwort erwähnt wird. (Elze 1897: 59)

Hier ist die tiefe Verbundenheit des Rezensenten und seine Vertrautheit mit der Tradition kroatischer Schriftlichkeit und ihres glagolitischen Zweiges zum Ausdruck gekommen. Es ist, als würde er sagen: „Die kroatische Übersetzung des Neuen Testaments, erst die wird etwas Richtiges sein.“

Obwohl die eindeutige Erklärung des Begutachters, dass Trubers Publikationen einwandfrei rechtgläubig seien, jede Behinderung seiner Arbeit aus dem Weg geräumt hatte, konnte sich dieser mit den im Gutachten vorgebrachten Einwänden nicht abfinden, sondern trat in eine Polemik darüber ein und verlangte, dass andere, seiner Meinung nach besser qualifizierte Begutachter darüber das letzte und maßgebliche Wort sprechen. In einem am 8. März aus Kempten an Christoph, den Herzog von Württemberg, gerichteten Brief stellt Truber den im anonymen Gutachten geäußerten Vorwürfen Folgendes entgegen:

Jch wil mit dem drucken, bis ich ein anders grundtlichs vnd glaubwirdigs judicium vber meine büecher von Creineren vnd Vntersteyreren vberkhume, styl stan. Der guet man, welcher sein judicium vber meine büecher der kü. w. vbergeben, jst kein Creiner noch Vntersteyrer, sonder ein Beßyakh, mag villeicht herr doctor Schalichius sein. Nun hab ich der kü. w. offenlich jn der epistula dedicatoria, dergleichen jn meinem sendschreiben an sie geschriben, das jch allein die Creiner, Vntersteyrer, Kherner, Histerreicher vnd die Windischen Märkheren, vnd nicht Beßyackhen, Crobaten, Behaim oder Poln zum arbitros vnd richter meiner geschrifften haben will. (Elze 1897: 62-63; Rajhman 1986: 55 und 56)

Der anonyme Begutachter beanstandet eigentlich, dass Truber slowenisch schreibt und Truber erwidert klar und deutlich, dass er eben einzig und allein slowenisch schreiben will. Er spricht seinem Rezensenten das Recht ab, über die Sprache seiner Schriften zu urteilen, weil er kein Slowene, sondern ein „Besjak“ ist, wie Truber die kajkavischen Kroaten nennt. Truber sagt nicht, woran er den Begutachter als „Besjaken“ erkennt, aber es ist leicht dessen Einwänden zu entnehmen, wodurch er als solcher erkennbar wird. Er betont insbesondere, dass Trubers Sprache denen, „die um Agram herum sind“, unverständlich sei, er nimmt weiterhin den Lautwert lateinischer Buchstaben, wie sie in ungarischer Schreibung verwendet werden, für selbstverständlich, und so haben sich ihrer auch die Kajkavier im damaligen Slavonien bedient, er bessert auch Wörter in krainerischer Lautung: semla, sa volo, perjatel, modri in kajkavisch kroatische: zemlja, za volju, prijatel, mudri aus. Und zu allem Überfluss zeigt er deutlich, dass ihn die kroatische (d. h. glagolitische) Übersetzung des neuen Testaments besonders interessiert, dass ihm an ihr - so könnte man sagen - mehr gelegen ist als an der slowenischen. Truber selbst jedoch hat im 
Vorwort zum ersten Teil der kroatischen Übersetzung des Neuen Testaments (Tübingen 1562) über die „Besjaken“ in Slavonien Folgendes geschrieben:

Die Sclauen (d. h. Slavonier) die man sonst Bessiacken nennt / haben fasst Vngerische vnd Crobatische Sitten vnd Eigenschafft / halten sich mit jrer Religion in allen dingen wie die Crobaten / allein das jre Priester lateinisch Meß lesen / vnd gehen weit walfarten. (Sakrausky 1989: 210)

Es war ihm daher gar nicht schwer, im Anonymus einen „Besjaken“ zu erkennen. Truber jedoch erkennt nicht nur die Herkunft seines anonymen Begutachters und lehnt dessen kritische Beurteilung seiner Schriftsprache ab, weil dieser kein Slowene ist. Er stellt auch eine Vermutung darüber an, wer dieser Begutachter sein könnte, und ist dabei so sicher, dass er nicht davon Abstand nimmt, diesen dem Herzog von Württemberg in schriftlicher Form zu nennen. Er ist geneigt zu glauben, dass es Paulus Skalich ist, eine besonders interessante, aber auch recht schillernde Persönlichkeit im Kreis der kroatischen Humanisten und Protestanten. Er war 1534 in Agram (Zagreb) als Sohn eines Lehrers bäuerlicher Herkunft, Mihalj Jelenčić, und der Katarina Skalić geboren. Nach dem frühen Tod seines Vaters ist seine Mutter mit dem Jungen nach Laibach (Ljubljana) gezogen, hat dort geheiratet und noch 1564 als Schneiderin gelebt. Auf Empfehlung des Laibacher Bischofs Urban Textor (Weber, Tkalec) wurde der damals dreizehnjährige „Paulus Skalich aus Agram“ als Stipendiat an die Wiener Universität aufgenommen und hat dort 1549 das Bakkalaureat, 1551 das Magisterium erlangt. In Bologna hat er 1552 als Zwanzigjähriger das Doktorat der Theologie erworben. Von dort ist er nach Rom gegangen und dann mit einem Empfehlungsschreiben des Papstes Julius III. zu König Ferdinand nach Wien zurückgekehrt. Dieser hat ihn als Hofkaplan angestellt und zum Koadjutor des Bischofs von Laibach auserwählt. Aber schon 1557 wurde er aus Wien vertrieben, angeblich wegen an der Universität gehaltener Vorträge und Dispute. Ferdinand, damals schon Kaiser, hatte ihm das sehr übel genommen. Die Zuneigung seines Sohnes Maximilian, der damals böhmischer König war, blieb ihm jedoch erhalten. Der König hatte protestantische Neigungen und hat den hochbegabten und vorzüglich humanistisch gebildeten jungen Mann voller Wissen und Begeisterung hoch geschätzt. Er hat ihm Empfehlungsschreiben gegeben und ein jährliches Einkommen gesichert. So ausgestattet begab sich Skalich nach Stuttgart, Heidelberg und Tübingen, wo er sich als Protestant bekannt und ein abenteuerliches Leben begonnen hat. Es ist ihm zwar nicht gelungen, sich dem mit südslawischen Bibelübersetzungen befassten Unternehmen zuzugesellen, er hat aber den Baron Hans Ungnad ganz für sich gewonnen. Auf Grund seines Familiennamens, den er von seiner Mutter hatte, hat er sich als Abkömmling des adeligen Geschlechts Scaliger aus Verona vorgestellt, der aus religiösen Gründen von seinen großen Besitzungen in Kroatien vertrieben worden war.

Nachdem er mit Ungnad einen Vertrag über den Rückerwerb seines Vermögens geschlossen hatte, in dem er ihm versprach, dass er ihm, sollte er ihm helfen, es zurückzugewinnen, die Hälfte davon überlassen werde, ging er zum Herzog von Preu- 
ßen, Albrecht, nach Königsberg. Dort wurde er allerbestens aufgenommen. Er hat den Herzog geradezu bezaubert. Dieser hat ihn zu seinem geheimen Berater ernannt und ihm das Herrengut Kreuzberg geschenkt. In Königsberg hat er sehr erfolgreich an der Universität gelehrt. Angehörige des preußischen Adels haben sich jedoch gegen ihn in Bewegung gesetzt und ihn öffentlich zum Abenteurer und Betrüger erklärt. Obwohl ihn der Herzog schützte, musste er nach Danzig (Gdańsk) fliehen und ist 1566 für geächtet erklärt worden. Darauf ist Skalich nach Berlin, Wittenberg und Paris gegangen. Später ist er nach Münster in Westfalen übersiedelt und hat dort seine Haushälterin geheiratet. Da hat er sich dann als Katholik bekannt und Schriften zu veröffentlichen begonnen, in denen er die Lehre der Römischen Kirche verteidigte. Er hat auch mit Hilfe des Bischofs von Münster erreicht, dass in Preußen das Urteil gegen ihn zurückgenommen wurde. Darauf ist er nach Danzig zurückgekehrt und hat von dort aus über die Rückgabe seiner preußischen Besitzungen verhandelt. Während dieser Verhandlungen ist er 1575 in Danzig gestorben und wurde dort in der Altstadt in der Karmeliterkirche begraben. Er hat eine Reihe bedeutender lateinischer Werke hinterlassen, die zum Teil seine persönlichen Verhältnisse zum Gegenstand haben, insbesondere seine erfundene adelige Herkunft, zum Teil die Lehre der Römischen Kirche in Frage stellen, zum Teil wiederum - die zuletzt geschriebenen - eben diese Lehre unterstützen. Von allen seinen Veröffentlichungen ist das Buch Encyclopaediae, seu Orbis disciplinarum, tam sacrarum quam prophanarum, Epistemon, Basel 1559 zweifellos die wichtigste. In diesem Buch wird das Wort Enzyklopädie zum ersten Mal in seiner heutigen Bedeutung verwendet. ${ }^{3}$

Diesen und einen solchen Paulus Skalich hat Truber also in seinem anonymen Begutachter erkannt. Es ist durchaus zuzugeben, dass seine Mutmaßung sehr überzeugend ist. Allerdings drängen sich dem Forscher dabei einige Bedenken im Hinblick auf topographische und chronologische Gegebenheiten auf. Bei der großen Entfernung zwischen Stuttgart beziehungsweise Tübingen und Wien wickelte sich der Vorgang der Begutachtung ungemein schnell ab. Skalich aber hat, zwei Jahre bevor in Wien die Beschaffung eines Gutachtens der Slowenischen Bibelübersetzung Trubers von König Maximilian erbeten wurde, Wien verlassen und ist nach Württemberg gezogen. Verständlicherweise sind da auch Zweifel an der Richtigkeit von Trubers Mutmaßung aufgekommen (vgl. Kidrič 1938: 37). Dennoch wird allgemein angenommen, dass er recht hat. Schließlich konnte Truber selbst, der mit Skalich in direkte Berührung und auch in Konflikt kam, am besten wissen, wo dieser sich zu der Zeit befand und ob er das anonyme Gutachten geschrieben haben konnte oder nicht. Allenfalls war dieser wohl der schriftgewandteste und sicherlich der gelehrteste „Besjak“ seiner Zeit, ein anerkannter Theologe und daher qualifiziert, die Rechtgläubigkeit von Trubers Druckwerken zu beurteilen. Darüber hinaus passt das anonyme Gutachten nicht nur durch seine „besjakischen“ Merkmale, sondern auch

3 Über Paulus Skalich vgl. Kukuljević (1875), Johannes Voigt (1848), Elze 1987: 62-63, Bučar 1910: 136-140. 
durch ihren gelehrten Schwung, ihre überlegene Beredsamkeit, den humanistischen Slavismus und nicht zuletzt durch einen Anflug von unseriöser Spiegelfechterei ganz vorzüglich zu dessen Charakter und Persönlichkeit. ${ }^{4}$ Man kann also ruhig davon ausgehen, dass Primus Truber in seinem Brief an Herzog Christoph vom 8. März 1560 wirklich mit Paulus Skalich polemisiert. Um so mehr, als ausdrücklich belegt ist, dass König Maximilian zu der Zeit mit ihm in Korrespondenz stand. ${ }^{5}$

Nachdem er ganz eindeutig erklärt hat, dass er eben für die Slowenen schreibt und für keine anderen Slawen, geht Truber zur Verteidigung seiner beanstandeten Schreibung über. Darüber schreibt er an den Herzog Folgendes:

So bin ich auch vergwist vnd wills beweisen, das meine orthographei peßer vnd leßlicher ist dan der Behaim, Poln vnd Beßyacken. Ein jeder Lateiner, Teütscher oder Walich kan meine geschrifften dermaßen lesen vnd aussprechen, das ein jedlicher windischer zuhörer mag die verstehen. Aber die behaimische vnd polnische geschrifft khan niemant dan sie lesen, dan sie setzen gar zuuil vnnotig consonanten jn ein wort oder sylben vnd schreiben vnzalpar sylben vnd gantze wörter on vocal. Sie setzen vnd pronuncieren c, wen schon nicht ein e oder i, sonder ein a oder o oder v nachgesetzt wirdet, für ein z. Darumb will mein judex fur zhlouik, das ist mensch, chlouik setzen; das wird kein Teütscher noch Lateiner nimmer fur zhlouik oder tschlouik, sonder fur klouik oder hlouik lesen etc. (Elze 1897: 63-64; Rajhman 1986: 55 und 56)

In einem anderen Brief, den er ebenfalls in Kempten am 19. März 1560 an den Laibacher Kirchenrat geschrieben hat, verteidigt Truber die deutschen Lehnwörter, die er in die von ihm begründete slowenische Schriftsprache einführt und die vom anonymen Begutachter beanstandet wurden. Darüber schreibt er in diesem Brief Folgendes:

[...] un mueß ein ersame landtschafft jn Crain, Vntersteyr vnd Karhenten vnd jre obrigkeit sambt euch das pöst thuen vnd der ku. w. grundtlich berichten, das der arbiter oder judex,

${ }^{4}$ Es ist vielleicht gut, auch ganz ausdrücklich zu betonen, dass hier auf eine Beurteilung der Person von Skalich und seiner Rolle im kroatischen Protestantismus und im südslawischen, eben slowenisch-kroatischen reformatorischen Buchdruck überhaupt nicht eingegangen wird. Skalich wird hier nur als bedeutende Erscheinung im europäischen intellektuellen Umfeld der zweiten Hälfte des 16. Jahrhunderts und als wichtige Persönlichkeit der kroatischen Kultur- und Literaturgeschichte betrachtet. Es wird daher überhaupt nichts zum Gegenstand der Polemik über die Verdienste von Skalich gesagt, die sich in dem abzeichnet, was Alojz Jembrih (1986: 129-139) und Rotar (1988: 84-88) darüber veröffentlicht haben. Was aber die grundlegenden und einfachen menschlichen Werte betrifft, kann man sich kaum gegensätzlichere Charaktere vorstellen als die von Truber und Skalich und, wenn man sie auf dieser Grundlage vergleicht, wird dieser Vergleich, wie immer man ihn wenden mag, immer zu Trubers Gunsten ausfallen.

5 Zu Trubers aus Kempten am 27. Juli 1560 geschriebenem Brief an König Maximilian wurde dazugeschrieben (Elze 1897: 93; Rajhman 1986: 76 und 77): Vnnd nachdem doctor Sthaclichius, mein gancz vertrauter gunstiger herr, auch die creinerische, besyackhische vnnd crabatische sprach vnnd die crabatische geschrifft zimlich schreiben vnnd lesen kan, e. ku. mt. welle jme schreiben vnnd auferlegen, daß er vnns auch jnn disem göttlichen werckh, welches er selbst für groß vnnd nutzlich achtet, behilflich sey. Rotar (1988: 80 f.) kommt zum überzeugenden Schluss, dass dies nicht von Truber selbst dazugeschrieben wurde und dass es von taktischen Rücksichten diktiert ist im Bestreben, den kroatischen protestantischen Buchdruck in Württemberg nicht zu gefährden. 
der sein vrtail vber meine büecher jrer w. zugestelt, vnrecht hab; dan die wörter, welche er verwirfft, werden auch von dem gemainen man, der nicht teutsch khan, jn Oberem vnd Vnterem Crain, vmb Cili, Windisch-Grätz gepraucht vnd verstanden. Jch will künen fur urshah vsroh, fur gnada milost, fur milost pomilosardyu, fur ferdamnene pogublene oli sgublene, fur trosht vtisshania, fur nuz prid, fur leben shivot, fur erbszhina dedina, fur lon plazhia, fur shtyma glas etc. setzen vnd dolmetschen, wie dan auch mich etlicher diser worter hab gepraucht. Aber ich hab bei der gemainer crainerischer sprach bleiben wöllen. Dergleichen mit der orthoigraphei hat auch großlich vnrecht. Es ist wahr, die Behaim prauchen das c offt fur z. Sie schreiben das wort zhlouik also ćlowik. Jch halte aber mein orthgraphei fur pößer dan jr, darumb werde ich dabei beleiben. (Elze 1897: 68; Rajhman 1986: 58 und 59-60)

Die Sache ist hier ganz klar dargestellt. Eine lateinische Schreibung für sein slowenisches reformatorisches Schrifttum entwerfend geht Truber vernünftig und gut begründet davon aus, dass die slowenische ethnische Gemeinschaft der deutschen zutiefst akkulturiert ist.

In seinem Vorwort zur kroatischen Übersetzung des Ersten Teils des Neuen Testaments (Tübingen 1562) sagt er das auch ganz ausdrücklich:

Der obern Windischen Ländern / gemeines Volck / [...] Wölche aber in Lands Crein / Vndersteyr vnd Kernten sitzen vnnd jr Wonung haben / die halten sich nach Art vnd Aigenschafft der Teutschen / klaiden sich auch auff teutsch / allein das die Weiber tragen besondere lange Schlayer am Kopff. (Sakrausky 1989: 210)

Es war daher am ehesten angebracht, sich mit der Schreibung der slowenischen Schriftsprache, die er durchaus bewusst und wohl durchdacht begründete, an die Tradition der deutschen Schriftlichkeit anzuschließen. Truber hat das gemacht und damit erreicht, dass jeder, der deutsch lesen kann, wenn er seine slowenischen Texte liest, diese auch annähernd richtig ausspricht, auch wenn er nur wenig Slowenisch kann. Da das eben im Entstehen begriffene slowenische literarische Umfeld in hohem Maße zweisprachig war, brachte das große Vorteile. Der Begutachter, der „Besjak“, gehörte hingegen einer anderen Tradition der Schriftlichkeit an. Daher auch sein Mangel an Verständnis für die Graphie der neuen slowenischen Übersetzungen. Truber weiß und bezeugt das, er unterrichtet uns geradezu darüber. Es ist das jene Tradition der Schriftlichkeit, die sich, wie er sagt, bei den Tschechen, Polen und Besjaken entwickelt und gefestigt hat. Er hätte hier nicht zuletzt auch die Ungarn erwähnen sollen, aber sein Gesichtskreis bleibt auf slawischsprachige Schriftlichkeit beschränkt. Diese andere Tradition, die bereits tief verwurzelte Gewohnheiten geschaffen hatte, widersetzte sich Trubers schriftsprachlichem Projekt und blieb ihm undurchlässig, so sehr die kajkavischen Kroaten sprachlich und auch kulturell den Slowenen nahe stehen. ${ }^{6}$ Sie widersetzte sich mit all jenem, das das mittelalterliche

\footnotetext{
${ }^{6}$ Neben diesem ausdrücklichen Zeugnis Trubers gibt es wirklich keine Grundlage für Voraussetzungen, dass er bei der Ablehnung der von Skalich gegen seine Schreibung vorgebrachten Einwände davon ausgehe, das „Besjakische“ des Skalich sei eine Sprache, in der nicht geschrieben wurde, eine solche also wie das Slowenische bis zu Trubers Werk, dass ihr kulturelles Niveau nur niedrig ist und nicht das Ansehen besitzt, das die Verwendung in Büchern einer Sprache verleiht. So Rotar (1988: 73): Skaliću sploh ne dopušča,
} 
kajkavische Slavonien, Szlovenszki orszag, wie es von seinen Schriftstellern genannt wird, in tiefer Verbundenheit mit der čakavisch stilisierten Schriftlichkeit der kroatischen glagolitischen Tradition verbindet. Diese Verbindungen bezeugt auch Truber selbst, indem er, wie schon oben angeführt, schreibt: Die Sclauen (d. h. Slavonier) die man sonst Bessiacken nennt / haben fasst Vngerische vnd Crobatische Sitten vnd Eigenschafft / halten sich mit jrer Religion in allen dingen wie die Crobaten (Sakrausky 1989: 210). Ersichtlich wird das auch daraus, dass der „Besjak“ statt sa volo, semla und modri unbedingt za volju, zemlja und mudri haben will und für die kroatische glagolitische Übersetzung des Neuen Testaments, das nach den hohen biblizistischen Anforderungen der reformierten evangelischen Kirche vorbereitet wird, mehr Interesse als für alles andere zeigt.

Trubers Polemik mit Skalich ist eigentlich sehr einfach. Alles liegt auf der Hand, wenn man nur die Gesichtspunkte bestimmt hat, von denen aus jede der beiden Seiten ihre Überlegungen anstellt und argumentiert. Es hat nur der eine überhaupt kein Verständnis für den Gesichts- und den Ausgangspunkt des anderen. Es gibt da nichts, was schwer zu ergründen oder ungesagt geblieben wäre. Interessant ist diese Polemik dadurch, dass sie ein unerwartet helles Licht auf die schriftsprachlichen

da bi posegal na to področje; gotovo ravna Trubar tako tudi zaradi dejstva, da se govorica „,teh, ki prebivajo okoli Zagreba“, torej Skalićeva govorica, sploh še ni povzpela na kulturno raven, ne dosega prestižne stopnje, ki jo označuje knjiga und S. 85: Kot smo videli, je Skalić skušal vsiljevati Trubarju govorico oziroma jezikovne značilnosti, ki izhajajo od tistih ljudi, ,, qui circa Zagrabiam sunt“. Torej je Skalić nasproti Trubarjevi knjižni promociji slovenščine ponujal govorico, ki se sploh še ni dvignila na kulturno raven knjižne rabe in veljave, tistemu Trubarju, ki se je tako zelo zavedal tega kulturnega primanjkljaja oziroma ki ga je bil pri Slovencih presegel po tako dolgotrajnem i skrbnem pretehtavanju, primerajoč pravopise raznih narodov. Das sind Rotars Überlegungen und es ist seine Rhetorik, keineswegs sind es aber die Gedankengänge Trubers. Dieser wusste sehr wohl, gerade deshalb, weil er die Graphien verschiedener Völker verglichen hatte, dass die „Besjaken“ darin einer weiteren und fest verwurzelten Tradition angehörten, welche die Schriftlichkeit mehrerer benachbarter Sprachen erfasst, und dass diese Tradition älter ist als die slowenische Literatur, deren Fundamente zu legen ihn eben beschäftigte. Er wusste auch gut, dass „Besjakisch“ schon zu seiner Zeit eine Sprache war, in der geschrieben wurde, und hat daher in seinen Vergleich auch dieses einbezogen. Das alles sagt er ja selbst. Er war jedoch der Meinung, und zwar nicht ohne Grund, dass diese Tradition nicht geeignet war, um auf ihr die slowenische Schreibung zu begründen. Auch war er an die deutsche Schreibung gewöhnt, ebenso wie Skalich an die ungarische, wie ROTAR (73) richtig bemerkt: To Trubarjevo izvirno pravopisno načelo pa Skaliću, vajenemu ogrske pisave med ,temi, ki žive okoli Zagreba“, torej ni bilo všeč. Das Schreiben nach deutscher graphischer Tradition schien Truber ganz natürlich deutlicher und verständlicher, besser lesbar - wie er sagt - zu sein. Eine Literatur, wie sie Truber damals bei den Slowenen begründete, gab es, als Skalich sein Gutachten schrieb, bei den Besjaken allerdings nicht, aber es gab, wie man gerade auch aus diesem Gutachten sieht, eine Schriftlichkeit mit ihrer Tradition und in ihr entstandene feste Gewohnheiten. Auch eine echte Literatur beginnt bei ihnen, wie man immer deutlicher erkennt, früher als man bis vor kurzem allgemein glaubte. Truber wusste das, hat es im anonymen Gutachten erkannt und hat dazu eindeutig Stellung bezogen. Er wusste, was er für das Slowenische wollte. Jene Gewohnheiten aber und jene Tradition waren fest und widerstandskräftig genug, um Trubers schriftsprachliches Projekt auf Dauer vom Szlovenski orszag fernzuhalten. 
Verhältnisse wirft, wie sie in der zweiten Hälfte des 16. Jahrhunderts im Bereich um die Grenze am Sutlafluss waren. Da diese politische Grenze schon seit Jahrhunderten zwar durchlässig und offen, aber auch beständig und fest gezogen war, ist bei aller sprachlichen Nähe des kajkavischen Kroatisch zum Slowenischen schon damals klar gewesen, auf welcher Seite sich die tragende Grundlage für die Entwicklung der slowenischen und auf welcher für die der kroatischen Schriftsprache befand (vgl. auch Rotar 1991: 95-102). Gerade dadurch, dass sie das deutlich zeigt, ist die Polemik des Primus Truber mit Paulus Skalich von nicht geringer Bedeutung und bleibt dauernd interessant.

\section{Quellen- und Literaturverzeichnis}

Bučar 1910:

Elze 1897:

Jembrih 1986:

Katičić 1997:

Kidrič 1938:

Kukuljević 1875:

Rajhman 1986:

Rotar 1988:

Rotar 1991:

Rupel 1965:

Sakrausky 1989:

Voigt 1848:
Franjo Bučar, Povijest hrvatske protestantske književnosti, Zagreb

Theodor Elze, Primus Trubers Briefe mit den dazugehörigen Schriftstücken, Tübingen

Alojz Jembrih, Pavao Skalić u službi reformacije, in: Družbena in kulturna podoba slovenske reformacije, ur. Darko Dolinar, Ljubljana, 129-139

Radoslav Katičić, Bilješka o polemici Primoža Trubara s Pavlom Skalićem, in: Studia in honorem Stephani Nyomárkay (= Hungaro-Slavica 1997), Budapest, 123-132

Francè Kidrič, Zgodovina slovenskega slovstva, Ljubljana Ivan Kukuljević, Pavao Skalić, Zagreb

Jože Rajhman, Pisma Primoža Trubarja, Ljubljana

Janez Rotar, Trubar in južni Slovani, Ljubljana

Janez Rotar, Die Nationwerdung der Slowenen und die Reformation. Trubars Benennungen von Ländern und Völkern, München

Mirko Rupel, Primus Truber. Leben und Werk des slowenischen Reformators, München

Oskar Sakrausky, Primus Truber. Deutsche Vorreden zum slowenischen und kroatischen Reformationswerk, Wien - Ljubljana Johannes Voigt, Paul Scalich, der falsche Markgraf von Verona, Berliner Kalender

Radoslav Katičić

Institut für Slawistik der Universität Wien

Universitätscampus AAKH, Hof 3

Spitalgasse 2, A-1090 Wien

radoslav.katicic@univie.ac.at 